\title{
Growth differentiation factor 15 contributes to cancer-associated fibroblasts-mediated chemo-protection of AML cells
}

Yuanmei Zhai ${ }^{1 \dagger}$, Jing Zhang ${ }^{1 \dagger}$, Hui Wang ${ }^{1 \dagger}$, Wei Lu' ${ }^{1}$, Sihong Liu' ${ }^{1}$, Yehua Yu' ${ }^{1}$ Wei Weng ${ }^{2}$, Zhiyong Ding ${ }^{3}$, Qi Zhu ${ }^{4}$ and Jun Shi ${ }^{*}$

\begin{abstract}
Background: Chemo-resistance is still a major obstacle in efforts to overcome acute myeloid leukemia (AML). An emerging concept has proposed that interactions between the bone marrow (BM) microenvironment and leukemia cells reduce the sensitivity of the leukemia cells to chemotherapy. As an important element of the tumor microenvironment, the cancer-associated fibroblasts (CAFs) are considered to be activated modulators in the chemo-resistance of many solid tumors. But their contribution to AML has yet to be fully understood. Here we report a critical role for CAFs which were thought to be a survival and chemo-protective factor for leukemia cells.

Methods: A retrospective study on the BM biopsies from 63 primary AML patients and 59 normal controls was applied to quantitative analysis the fiber stroma in the BM sections. Then immunohistochemistry on the BM biopsies were used to detect the makers of the CAFs. Their effects on drug resistance of leukemia cells were further to be assessed by co-cultured experiments in vitro. Moreover, the possible mechanisms involved in CAF-mediated chemo-protection of AML cells was investigated by antibody neutralization and siRNA knockdown experiments, with particular emphasis on the role of GDF15.

Results: In our study, excessive reticular fibers in the BM led to higher frequency of relapse and mortality in primary AML patients, bringing the inspiration for us to investigate the functional roles of the fiber-devied cells. We declared that the CAF cells which expressed higher levels of FSP1, a-SMA or FAP protein were widely distributed in the marrow of AML. Then in vitro co-cultured tests showed that these CAFs could protect leukemia cell lines (THP-1/K562) from chemotherapy. Interestingly, this effect could be decreased by either treatment with a neutralizing anti-GDF15 antibody or knockdown GDF15 (with siGDF15) in CAFs. Furthermore, we also confirmed that the GDF15 ${ }^{+}$cells mainly co-localized with FAP, which was identified as the typical phenotype of CAFs in the BM stroma.
\end{abstract}

Conclusions: We firstly demonstrate that the functional CAFs are widespread within the BM of AML patients and should be a critical chemo-protective element for AML cells by producing amount of GDF15.

Keywords: Bone marrow microenvironment, CAFs, Acute myeloid leukemia, GDF15

\footnotetext{
* Correspondence: junshi@sjtu.edu.cn

${ }^{\dagger}$ Equal contributors

'Department of Hematology, Shanghai Jiao Tong University Affiliated Sixth

People's Hospital, Shanghai 200233, China

Full list of author information is available at the end of the article
} 


\section{Background}

Chemo-resistance remains a significant challenge in acute myeloid leukemia (AML) and is one of the critical prognostic elements. Evidence has suggested that the interaction between leukemia cells and the BM stroma could affect the chemo-sensitivity of the tumor cells [1]. As an important element of the tumor microenvironment, fibroblasts with an activated phenotype, referred to as 'activated myofibroblasts' or 'cancer-associated fibroblasts' (CAFs), have been reported to play a critical role in the chemo-resistance of solid tumors [2]. However, CAFs still appear not to be a concern in leukemia because BM biopsies are not routinely performed in AML patients. Therefore, whether CAFs exist in the BM microenvironment and whether they are involved in resistance to chemotherapy still remain unclear.

CAFs display phenotypes that are similar to those of myofibroblasts derived from quiescent fibroblasts, which are activated when they interact with carcinoma cells during tumorigenesis [3]. These powerful cells are immunologically defined primarily by the expression of $\alpha$-smoothmuscle actin ( $\alpha$-SMA), fibroblast-activated protein (FAP), and fibroblast-specific protein 1 (FSP1) [4-6]. Datas have revealed that CAF cells are involved in the migration, growth and chemo-resistance of tumor cells in several solid tumors, including human prostate, pancreatic, colorectal carcinoma, and in hematologic malignancies, such as multiple myeloma, and the function mainly by secreting a variety of cytokines [7-9]. In the BM from mouse models of acute lymphoblastic leukemia (ALL), we have previously determined a protective niche, which is dynamically transient between Nestin ${ }^{+}$and $\alpha-\mathrm{SMA}^{+}$cells,causing the production of reticular fibers in response to chemotherapy. CAF-like cells and fibers may provide chemoresistance for leukemia cells in vivo. Meanwhile, leukemia cells contribute to the protective niche formation by secreting chemotactic factors and growth factors, in particular, growth differentiation factor 15 (GDF15) [10]. GDF15, a divergent member of the TGF- $\beta$ superfamily, is highly expressed only in the placenta and in macrophage cells under physiological conditions [11]. However, it is clinically associated with disease progression in numerous tumors [12-14]. Recently, it has been demonstrated that CAFs could be a novel source of GDF15 in human prostate cancer and multiple myeloma [7, 15]. We propose that CAFs within the BM of AML could also counter the chemo-sensitivity of leukemia cells by producing GDF15.

In the current study, excessive reticular fibers in the BM led to a poor prognosis in primary AML patients, making it important to investigate the functional roles of the fibroblast cells. After demonstrating the presence of CAFs in the marrow microenvironment of primary AML and their ability to protect leukemia cells from chemotherapy agents in vitro, we assessed whether GDF15 contributed to the CAF-mediated chemo-protection of AML cells either by using a neutralizing anti-GDF15 antibody or by knocking down GDF15 (siGDF15) in the CAFs as well as by investigating the distribution of GDF15 in the cytoplasm of CAFs within BM sections from AML patients. These findings were used to investigate the functional roles of CAFderived GDF15 in chemo-resistance of leukemia and whether CAF cells, by producing GDF15, could be a survival and chemo-protective element for leukemia cells.

\section{Methods}

\section{Primary AML samples and normal controls}

Primary AML patients except acute promyelocytic leukemia $(n=63)$ were prospectively enrolled in our study after providing written informed consent. Cases of newly diagnosed lymphoma patients without bone marrow involvement were selected as the normal control group $(n=59)$. There were no statistical differences in age and sex between these two groups (data were not shown). The studied patients with AML received the induction/reinduction chemotherapy protocols and all the BM trephine biopsy specimens and BM aspirates were obtained from Shanghai Jiaotong University-affiliated hospitals (Shanghai, China). The patients were diagnosed according to the FAB classification. Complete remission (CR) and relapse were diagnosed according to Cheson et al [16]. And the refractory AML cases were defined in accordance with Schmid et al [17]. The AML patient characteristics are presented in Table 1 . The pathologists who examined the BM samples were not participant in this study and were innocent about the group situation.

\section{Cell culture and regents}

Mesenchymal stem cells (MSCs) were obtained from the $\mathrm{BM}$ of primary AML patients after informed consent. The marrow was diluted twice with phosphate buffered saline

Table 1 Patient demographics and clinical characteristics

\begin{tabular}{llll}
\hline Characteristic & $\begin{array}{l}\text { Refractory group } \\
(n=25)\end{array}$ & $\begin{array}{l}\text { CR group } \\
(n=38)\end{array}$ & $P$ value \\
\hline Age (years) & 53 & 60 & 0.261 \\
Median age & $23-82$ & $18-80$ & \\
Range & $18 / 7$ & $27 / 11$ & 0.935 \\
Sex (Male/Female) & - & - & 0.709 \\
FAB subtype & 1 & 1 & \\
M0 & 1 & 6 & \\
M1 & 8 & 10 & \\
M2 & 8 & 15 & \\
M4 & 5 & 4 & \\
M5 & 1 & 1 & \\
M6 & 1 & 1 & \\
M7 & & & \\
\hline CR comprete & & & \\
\hline
\end{tabular}

$C R$ complete remission, $F A B$ French-American-British Classification 
(PBS), and then isolated by Ficoll-Hypaque (Axis-Shield Diagnostics, Dundee, Scotland, UK) density-gradient centrifugation. Monocytes were collected by adherence to a plastic flask and incubated for $48 \mathrm{~h}$ in MesenCult ${ }^{\circ}$ medium (STEMCELL Technologies, Vancouver, BC, Canada). The phenotype and multipotential differentiation of MSC cells used in the study has been verified (Additional file 1: Figure S1-2). Then BM-MSCs were treated with recombinant human TGF- $\beta 1$ (10ng/ml, R\&D Systems, Minneapolis, MN, USA) to trigger CAF differentiation. Conditioned medium from the CAFs or MSCs was obtained from cells cultured in regular medium with $1 \%$ FBS. The leukemia cell lines THP-1 and K562 (American Type Culture Collection, Manassas, VA, USA) were cultured in 1640 or low-glucose DMEM supplemented with $10 \%$ fetal bovine serum (FBS) and penicillin-streptomycin (Gibco, Grand Island, NY, USA) at $37{ }^{\circ} \mathrm{C}$ in $5 \% \mathrm{CO}_{2}$. Additionally, where appropriate, the leukemia cells were pre-incubated with the TGF- $\beta 1$ inhibitor SB431542 (Sigma, St Louis, MO, USA) for $2 \mathrm{~h}$ at 37 ${ }^{\circ} \mathrm{C}$ in $5 \% \mathrm{CO} 2$.

\section{Transwell co-culture of CAFs with leukemia cells}

CAF cells $\left(2 \times 10^{4}\right.$ initial cell count) were cultured for 24 $h$ in the upper side of a transwell chamber partitioned by a polycarbonate membrane (8.0um pore size, Corning Incorporated, Costar). And $2 \times 10^{5} \mathrm{THP}-1$ and $\mathrm{K} 562$ cells were co-cultured in the lower part in 1640 or DMEM medium with $10 \% \mathrm{FBS}$ at $37^{\circ} \mathrm{C}, 5 \% \mathrm{CO}_{2}$ for $48 \mathrm{~h}$.

\section{Evaluation of leukemic cell viability}

THP-1 and K562 cells from suspension cultured, direct cocultured or transwell co-cultured groups were treated with Ara-C (10uM, TCI Company, Tokyo chemical industry, Tokyo, Japan) for $48 \mathrm{~h}$. Directly co-cultured THP-1/K562 cells were separated from the MSCs or CAFs monolayer by careful pipetting. After the leukaemic cells were collected, the monolayer was observed under the microscope $(100 \times)$ to confirm that the monolayer was not damaged and that fewer than 10 leukaemic cells/vision field remained attached. Cell viability was evaluated by trypan blue exclusion in triplicate samples and quantified by a Cellometer Mini (Nexcelom, Lawrence, MA, USA).

\section{Immunohistochemistry and the reticulin fiber density (RFD) assay}

The BM tissue samples were used for immunohistochemistry and Gomori's stain. The rabbit anti-FSP1/S100A4 monoclonal antibody (Millipore, Billerica, MA, USA), rabbit anti-FAP polyclonal antibody (Abnova, Taipei, Taiwan), goat anti- $\alpha$-SMA polyclonal antibody (Abcam, Cambridge, MA, USA), rabbit anti-GDF15 monoclonal antibody (Cell Signaling, Danvers, MA, USA), mouse antiVimentin monoclonal antibody (Santa Cruz, Dallas, CA, USA), and mouse anti-CD68 monoclonal antibody
(Abcam) incubations were performed according to the manufacturer's instructions. Images of the slides stained for these markers were scanned at $40 \times$ magnification using the optical microscope (Olympus Co., Tokyo, Japan). The positive rate of each marker was quantified by counting the ratio of the positive cells in all nuclear cells. The reticulin fiber density (RFD) of the samples was assessed according to Norén-Nyström et al [18]. All readings and estimations were performed in a blinded manner.

\section{Flow cytometry}

The Alexa Fluorescence488 (AF488)-conjugated monoclonal antibodies specific for $\alpha$-SMA (R\&D Systems, Minneapolis, MN, USA), a rabbit anti-FAP polyclonal antibody (Abcam, Cambridge, MA, USA) and a FITC-conjugated anti-rabbit polyclonal antibody were used (eBioscience, San Diego, CA, USA). The appropriate isotype-matched antibodies were used as controls (R\&D Systems). For the apoptosis assay, THP-1 and K562 cells $\left(5 \times 10^{5}\right)$ were stained with Annexin-V and propidium iodide (PI) for apoptosis detection (BD, Franklin Lakes, New Jersey, USA) according to the recommended protocol on a Becton Dickinson Flow Cytometer. For cell cycle analyses, THP-1 cells were treated with RNase A and PI (Sigma, St Louis, MO, USA). The measurements were made using a flow cytometer (Beckman, Urbana, IL, USA).

\section{Quantitative reverse transcription PCR (qRT-PCR)}

Total RNA was extracted using Trizol (Invitrogen, Paisley, UK), and the RNA was converted into cDNA using the Primer Script TM RT reagent Kit (Takara Bio Inc, Otsu, Shiga, Japan). All real-time PCR reactions were performed using an ABI 7500 real-time PCR system (Biosystems, Foster City, CA, USA) and the SYBR Premix Ex Taq reagent kit (Takara Bio Inc).

\section{Western blotting and ELISA}

MSC or CAF cells were harvested in a radioimmunoprecipitation assay buffer for western blotting analysis (Beyotime, Haimen, Jiangsu, China). Mouse anticollagen I monoclonal antibody (Abcam, Cambridge, MA, USA), rabbit anti-collagen III polyclonal antibody (Abcam), rabbit anti-FAP polyclonal antibody (Abnova, Taipei, Taiwan), rabbit anti- $\alpha$-SMA monoclonal antibody (Epitomics, Burlingame, CA,USA), rabbit antiGDF15 polyclonal antibody (Bioworld, Louis Park, MN,USA) and mouse anti- $\beta$-actin monoclonal monoclonal antibody (Cell Signaling, Danvers, MA, USA) were used. Antigen detection was performed using a goat anti-rabbit or an anti-mouse IgG secondary antibody (Cell Signaling) conjugated to HRP and an ECL western blotting substrate (Pierce, Waltham, MA, USA). The BM aspirates were collected from newly diagnosed AML patients which were divided into CR 
group $(n=9)$ and refractory group $(n=8)$ and normal controls $(n=11)$. The ELISA analysis for GDF15 was performed according to the manufacturer's instructions (R\&D systems).

\section{RNA interference}

GDF15 siRNA and the control siRNA products were purchased from Gene Pharma Company (Suzhou, Jiangsu, China). Transfection of siRNA was performed using Lipofectamine2000 (Panc05.04) or Lipofectamine RNAiMax (Invitrogen, Paisley, UK) following the manufacturer's protocol. The validation of target gene knockdown was performed using qRT-PCR using the delta-delta CT method.

\section{Statistical analyses}

A Mann-Whitney U test was applied to compare the RFD of the AML patients and the normal controls. The statistical analyses were performed using the two-sample two-tailed Student's t-test. The survival curves were constructed using the Kaplan-Meier method, and the logrank test was used to test the difference in RFS and OS between cases with different RFD values. RFS was defined as the time between diagnosis and the onset of the first relapse, and OS was defined as the time between diagnosis and the occurrence of death or when lost to follow-up. Independent prognostic parameters for OS and RFS were identified by univariable Cox regression analyses and a multivariable Cox regression model using backward elimination with an exclusion significance level of $5 \%$. The data are described as the mean \pm standard deviation (SD), and $p$ values $\leq 0.05$ were considered significant. All statistical analyses were conducted by using the SPSS 18.0 software program (SPSS, Chicago, IL, USA).

\section{Results}

\section{Characteristics of the patients}

The median ages of patients in the refractory group $(n=$ $25)$ and CR group $(n=38)$ were 53 years (range: 2382years) and 60 years (range: $18-80$ years) respectively. The ratios of male/female in these two groups were 18/7 and 27/11 respectively (Table 1 ). There were no statistical differences in age, sex and FAB subtypes between the two groups.

\section{RFD is higher in AML and correlates with the chemotherapy outcome}

Some data have indicated that increased fibrosis in the $\mathrm{BM}$ is a physical barrier against the effective delivery of therapeutic agents in childhood acute lymphoblastic leukemia (ALL) [19]. In our study, Gomori staining showed that abnormally proliferated reticular fiber, shown as black silk filaments, was distributed more densely in refractory AML patients (Fig. 1a). The RFD score in the refractory patients was higher when compared to the CR group, and both of them were significantly higher than the control (Fig. 1b). Furthermore, the Cox's regression model showed that only the factor of RFD was associated with higher risk of relapse and death $(P=0.029$ and 0.000 , respectively) (Table 2$)$. This was further illustrated in a Kaplan-Meier survival analysis. The cutoff used for low and high RFD was decided by exploring RFD data in Cox's analyses dividing the material by the median and quartiles. In the AML-CR group, there was a poorer prognosis in RFS for cases with RFD $>1.13$ \% (Fig. 1c). And in all the AML patients, we also concluded that patients with RFD score of more than $3.68 \%$ revealed a shortened overall survival (Fig. 1d). However, there was no correlation between the RFD score and blasts either in the peripheral blood or in the BM (Figs. 1e-f), suggesting that the fibers neither anchor the leukemia cells in the BM nor promote blast proliferation. Therefore, we hypothesized that the fibers may not directly affect AML patient prognosis, although the RFD score indicated a poor outcome in AML patients. Based on this information, we focused on the reticulin fiber-derived cells.

\section{The reticulin fibers were mainly derived from the CAFs in AML}

The fibers in the BM were derived mainly from mesenchymal cells which were a heterogeneous population mainly comprised of adipocytes, macrophages and fibroblasts [20-22]. As we all know that adipocytes are seldom found in the BM of primary AML, therefore, adipocytes were not assessed in the survey. The mesenchymal markers Vimentin, FSP1, $\alpha$-SMA and FAP were immunoassayed to identify fibroblasts, and macrophage cells were identified by CD68. As illustrated in Fig. 2, Vimentin, as a common marker of mesenchymal cells, showed no difference in the BM from AML patients and controls, indicating that the increased fibers in AML may originate from some special subpopulations of mesenchymal cells. However, there represented no difference in the positive rate of CD68 in the BM between these two subgroups, indicating that macrophages did not contribute to fibrosis in AML. Among the other mesenchymal markers, there was a significant increase in the cells positive for FSP1, $\alpha$-SMA or FAP in the AML samples compared to the controls, and these are common markers of CAFs [23]. We thus hypothesis that the amount of CAFs strongly contributed to the increased fibers in AML, and we further to explore whether CAFs could play particular functions in AML progression.

\section{CAFs protect leukemia cells from chemotherapy}

We focused on the protective effects of the CAFs in the leukemia cells from chemotherapy. As illustrated in 


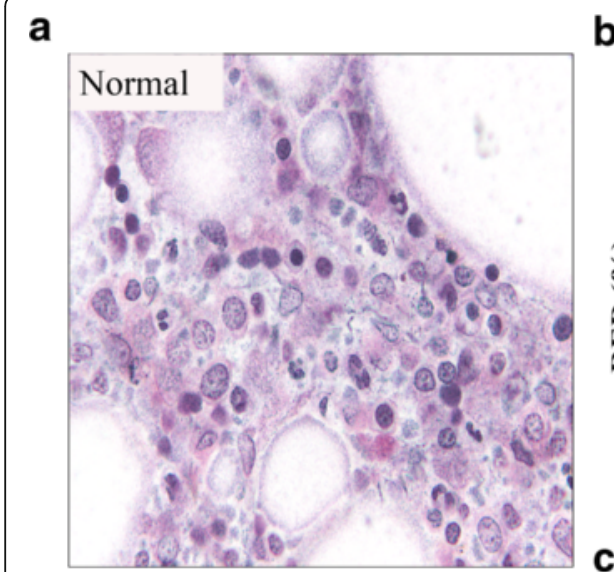

b
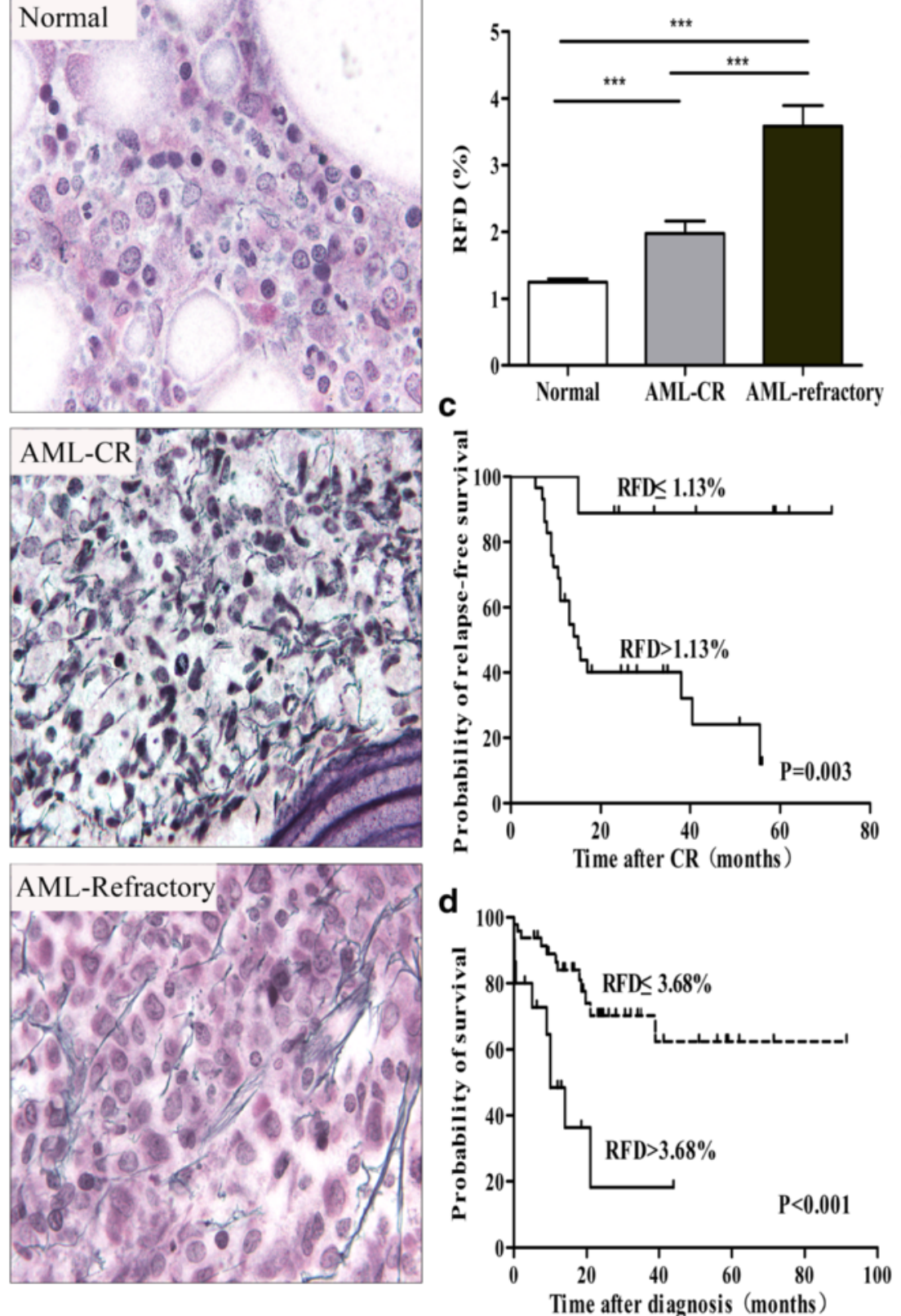

months) e
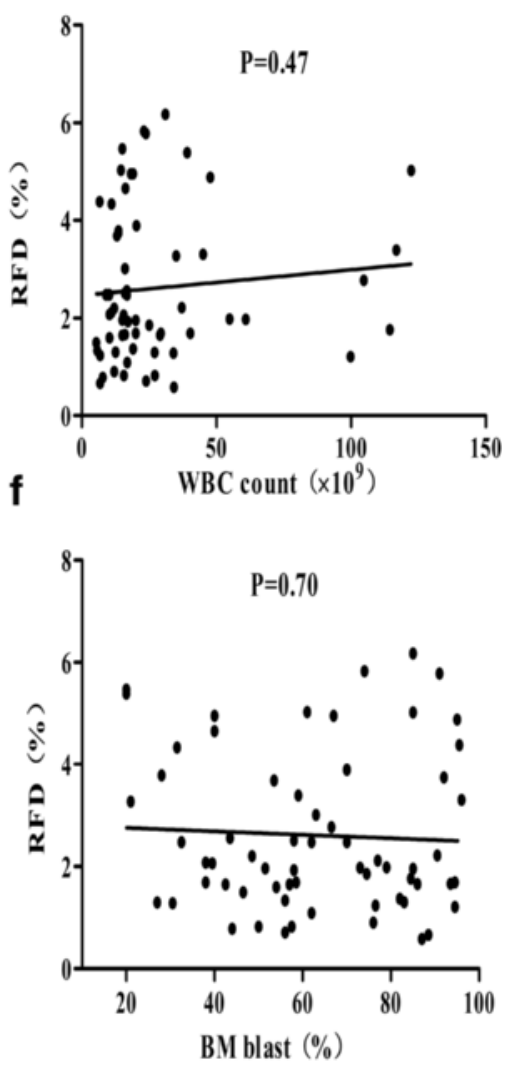

Fig. 1 The RFD score is higher in AML and is related to outcome. a Examples of the Gomori staining of reticulin fibers in the BM sections from normal controls and AML patients, which were further divided into CR and refractory groups. Both images are at a magnification of 1000x. $\mathbf{b}$ The RFD for the AML-CR $(n=38)$ and AML-refractory $(n=25)$ patients compared to the normal controls $(n=59)$, ${ }^{* * *} p<0.001$ (c) RFS of AML-CR patients subdivided into high $(>1.13 \%)$ and low ( $\leq 1.13 \%)$ RFD groups, $P=0.003$. d OS of all the AML patients subdivided into high ( $>3.68 \%)$ and low ( $\leq 3.68 \%)$ RFD groups, $P<0.001$. e-f Scatter plot: RFD vs. blasts in the peripheral blood and the blasts in the BM of primary AML patients using linear regression analysis $(P=0.47$ and 0.70 respectively)

Fig. 3a-c, under TGF- $\beta 1$ treatment, the stroma cells exhibited a larger volume and an increase in cytoplasmic content and uniformly expressed $\alpha$-SMA and FAP on the cell membrane as assessed by flow cytometry. These cells also had the capacity to synthesize higher levels of collagen I, collagen III, $\alpha$-SMA and FAP protein as measured by western blotting, which further confirmed that MSCs could be transformed into CAF cells by TGF- $\beta 1$. Co-cultured experiments showed that the mean survival of the CAF co-cultured leukemia cells was significantly 
Table 2 Cox regression for overall survival (OS) and relapse-free survival (RFS)

\begin{tabular}{|c|c|c|c|c|c|c|c|c|}
\hline \multirow[b]{2}{*}{ Variable } & \multicolumn{4}{|c|}{ RFS } & \multicolumn{4}{|l|}{ OS } \\
\hline & $n$ & $\mathrm{HR}$ & $95 \% \mathrm{Cl}$ & P & $n$ & $\mathrm{HR}$ & $95 \% \mathrm{Cl}$ & $P$ \\
\hline PWBC $\left(10^{9} / \mathrm{L}\right)$ & 38 & 1.009 & $0.988-1.030$ & 0.420 & 63 & 1.003 & $0.988-1.019$ & 0.674 \\
\hline Age (years) & 38 & 0.950 & $0.974-1.025$ & 0.950 & 63 & 1.002 & $0.976-1.030$ & 0.862 \\
\hline BMP (\%) & 38 & 0.987 & $0.965-1.010$ & 0.275 & 63 & 0.990 & $0.971-1.010$ & 0.330 \\
\hline RFD (\%) & 38 & 1.400 & $1.035-1.892$ & 0.029 & 63 & 1.755 & $1.331-2.312$ & 0.000 \\
\hline
\end{tabular}

Abbreviations: PWBC peripheral white blood cell, BMP Bone marrow precursors, RFD reticulin fiber density, HR hazard ratio, Cl confidence interval

increased compared with the cells co-cultured with the MSC or cultured alone (Fig. 3d). Leukemia cells cocultured with the CAFs exhibited less apoptosis as determined by AnnexinV/PI staining analysis (Fig. 3f), suggesting that CAF reduce the sensitivity of the leukemia cells to chemotherapeutic agents. Moreover, the cell cycle status of the residual leukemia cells revealed an increased number of cells which arrested in the G0/G1 phase, indicating that the CAFs protected the leukemic cells by maintaining them in a quiescent phase thus decreasing their chemotherapy sensitivity (Fig. 3e). To verify the effect of soluble factors secreted by CAFs on the chemotherapy sensitivity of the leukemia cells, transwell experiment was carried out to prevent direct contact but permit exchange of soluble diffusible factors. As shown in Fig. 3g, the viable cell numbers in the transwell system were significantly increased when compared to the suspension cultured subgroup $(p<0.001)$. Furthermore, when the leukemia cells cultured in the CAF-CM as described in the methods above, the viability of the

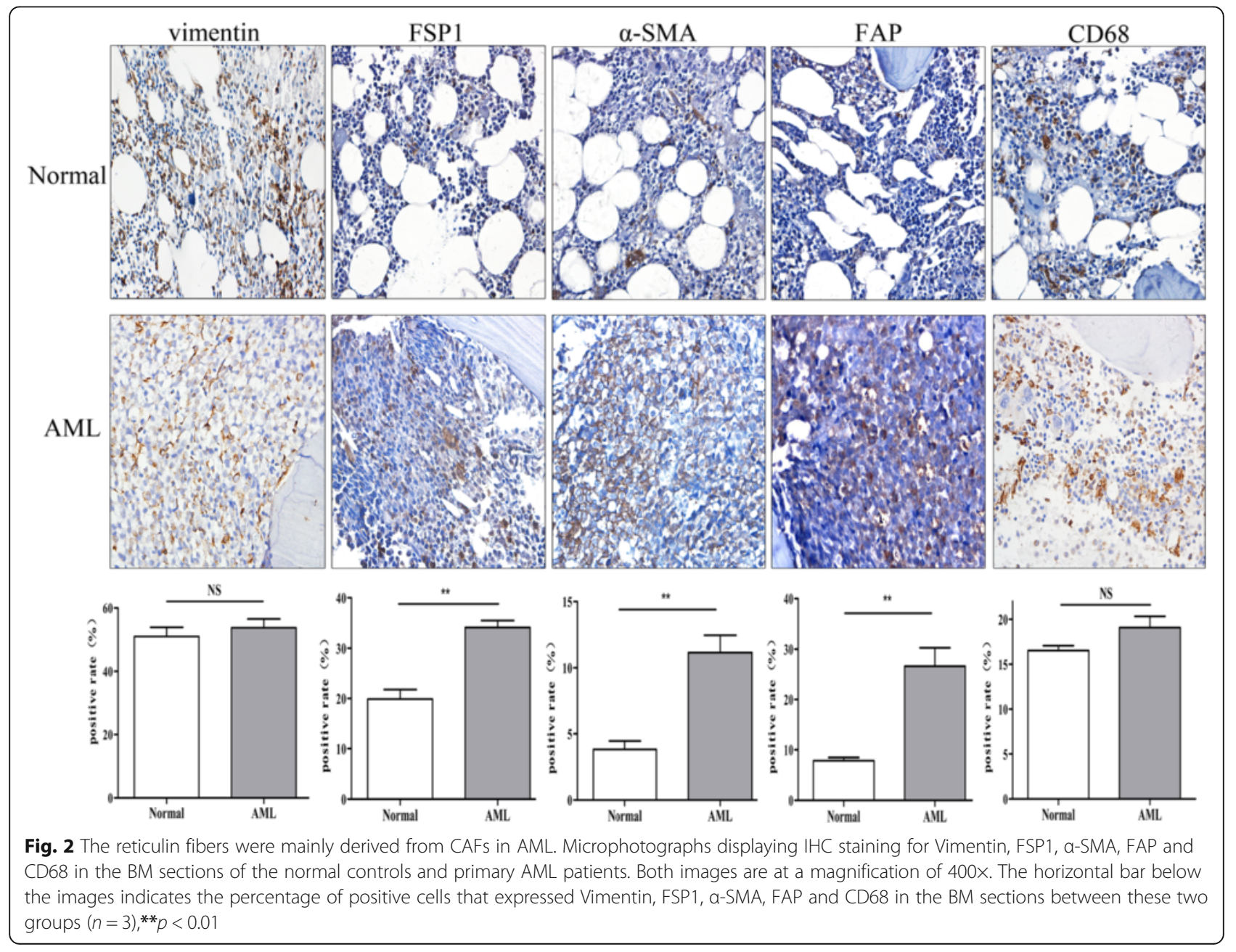




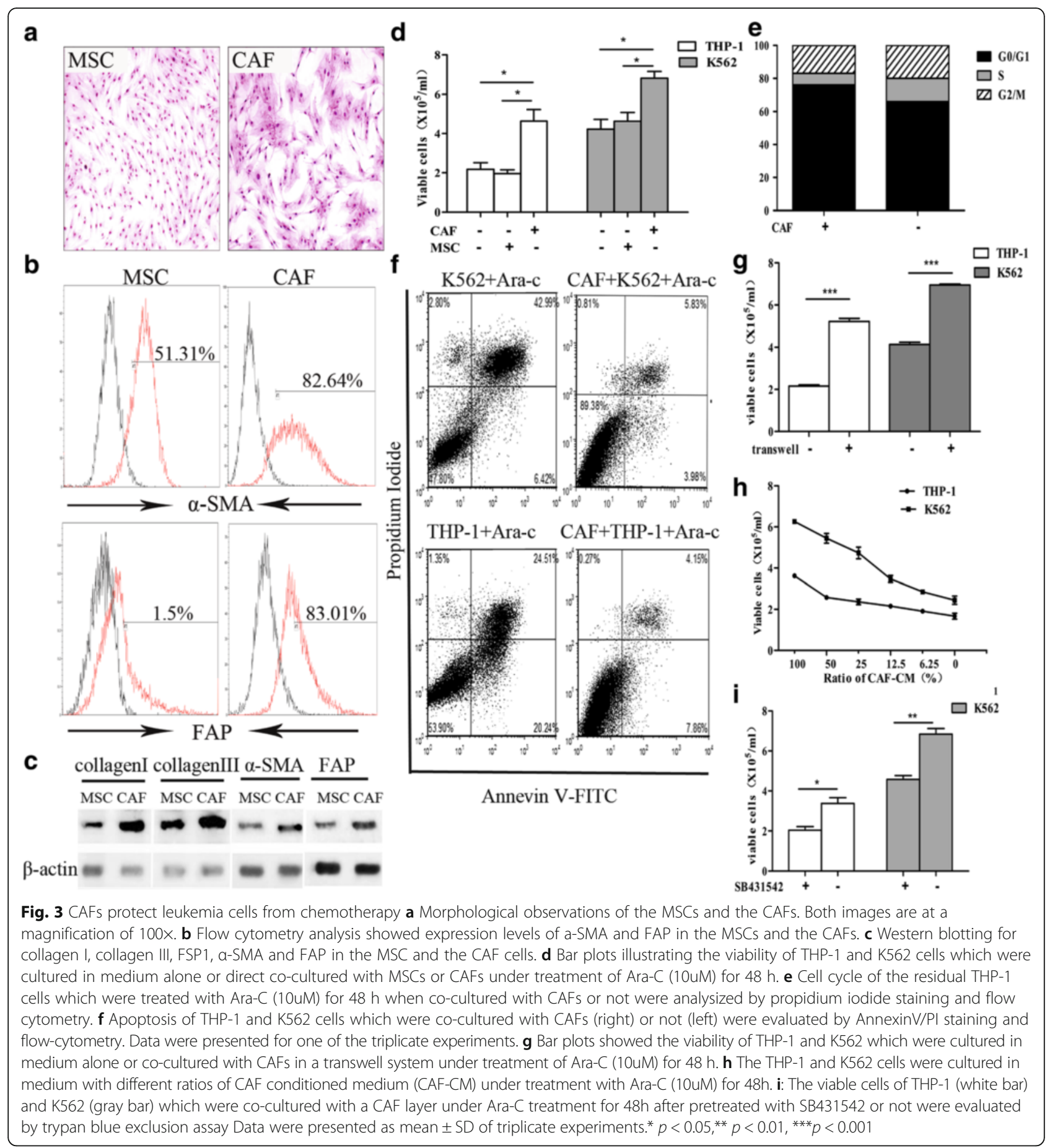

leukemia cells was decreased when the ratio of the CAFCM was reduced (Fig. 3h). SB431542, which is considered to be a TGF- $\beta$ receptor (ALK4/5/7) inhibitor, could partly abolished the protective effects of the CAFs for the tumor cells from chemotherapy (Fig. 3i), indicating that the chemo-protection for AML cells from CAF was mediated by some soluble substances, most likely TGF- $\beta$ superfamily.
GDF15 contributes to the CAF-mediated chemoprotection of AML cells

Based on our previous results showing that GDF15 was markedly up regulated in the residual leukemic cells after chemotherapy [10] and reports that GDF15 confers drug resistance to other cancer cells [24-27], we questioned whether CAFs in the BM could also play an important role by secreting GDF15. As shown in Fig. 4a-c, the GDF15 in 

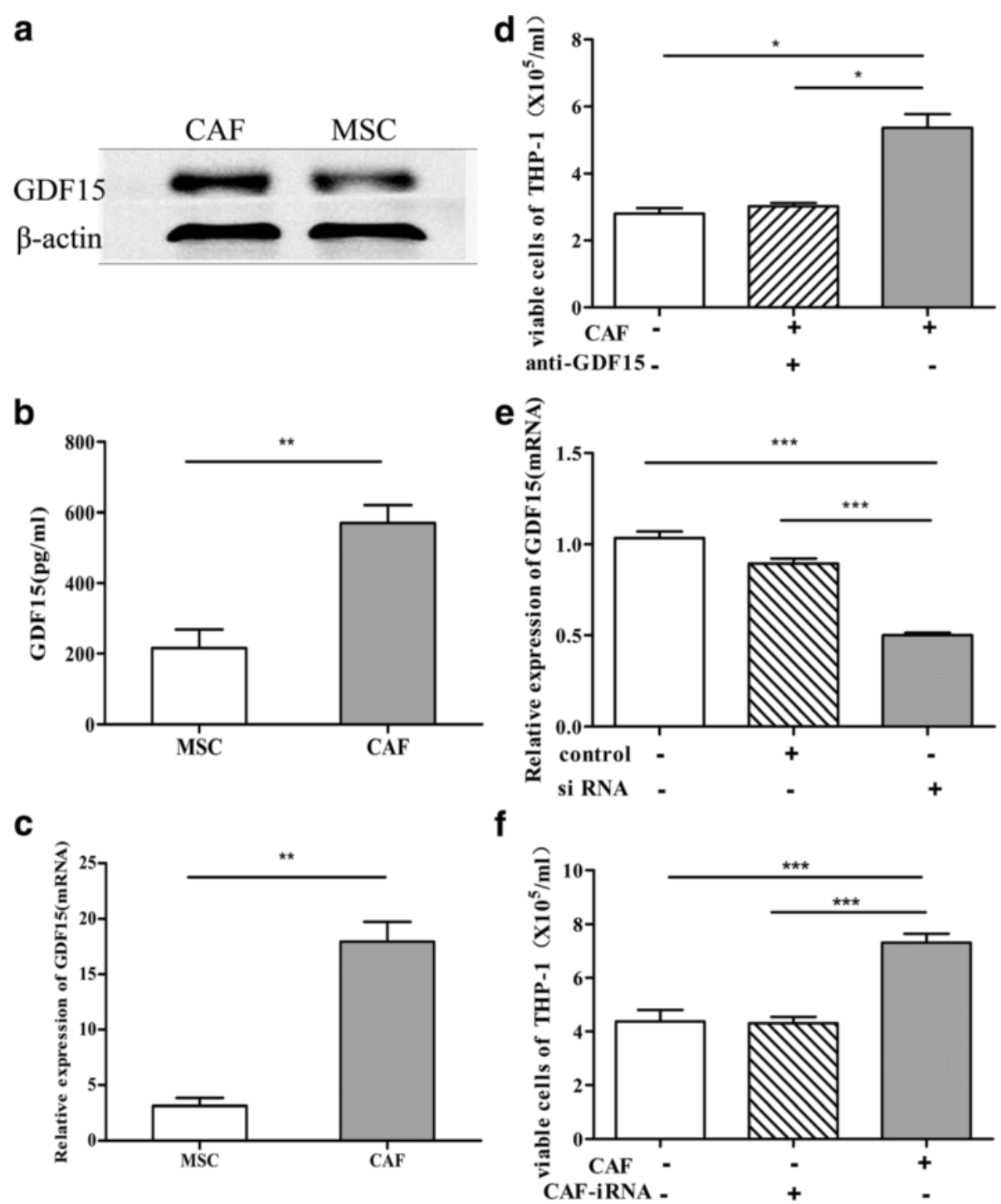

Fig. 4 GDF15 contributed to the CAF-mediated chemo-protection of the AML cells. (a-c) Western blot, ELISA and Quantitative RT-PCR analyses represented GDF15 expression in the MSC and the CAF cells. $\mathbf{d}$ The bar plots showed the relative viability of the THP-1 cells cultured in medium alone or co-cultured with CAFs added with or without an anti-GDF15 antibody under the treatment of Ara-c (10uM) for 48 h. e Quantitative RTPCR showing GDF15 expression in the CAFs after nucleofection of a GDF15-specific siRNA or a non-targeting control siRNA for $48 \mathrm{~h}$. $\mathbf{f}$ The number of viable THP-1 cells cultured in medium alone or direct co-cultured with the layer of CAFs after nucleofection of GDF15-siRNA or not under the treatment of Ara-C (10uM) for $48 \mathrm{~h}$. The data are the result of three independent experiments and are presented as means $\pm \mathrm{SD}^{*} p<0.05$, ${ }^{* *} p<0.01,{ }^{* * *} p<0.001$

the cell lysates of the CAFs was significantly increased when compared to MSCs and was further abundantly released into the culture supernatant, confirming that the CAFs secreted enough GDF15. To further investigate the functional roles of GDF15, the neutralizing anti-GDF15 antibody was added to the co-cultured system. As shown in Fig. 4c, under the treatment of Ara-c and the neutralizing anti-GDF15 antibody, the survival of THP-1 was decreased. In addition, we knocked down GDF15 in the CAFs with a small interfering RNA targeting GDF15
(siGDF15) and then validated by RT-PCR analysis (Fig. 4d). As shown in Fig. 4e, these CAF cells nearly lost their potential to protect THP-1 cells from chemotherapy. Our results demonstrating that GDF15 substantially contributes to the CAF-mediated chemo-protection of AML cells.

\section{GDF15 in the BM of AML patients}

To further confirm the pathophysiological contribution of GDF15 in CAFs, the amount of GDF15 in the BM aspirates was compared between the normal controls and 
the primary AML patients. To avoid the confounding effects of therapy-induced elevations of GDF15, the BM aspirates were derived from treatment-naïve patients. As shown in Fig. 5a, GDF15 in the BM aspirates from AML-refractory group was higher than the AML-CR group and both of them were significantly higher than the control group. To further investigate the distribution of GDF15 in the BM, immunohistochemistry analyses were performed in the BM biopsies. As illustrated in Fig. 5b, GDF15 was constitutively expressed in the cytoplasm of spindle cells, which were clearly observed in the BM biopsies from the AML patients, whereas these cells were scarcely in the controls. In addition, immunoassays on the serial sections showed that these GDF $15^{+}$ cells were mainly co-localized with FAP, which was identified as the typical immunophenotype of the CAFs (Figs. 5c). These results confirmed the possibility that GDF15 contributes to the CAF-mediated chemotherapy protection for leukemia cells.

\section{Discussion}

As it is known that the normal tumor microenvironment becomes 'corrupted' during tumor development, which is reflected by appearance of a large and heterogeneous category of cancer-associated fibroblasts (CAFs) [28]. CAF cells are considered active modulators of the tumor microenvironment among many solid tumors [29-31]. However, few studies have directly addressed the role of the CAFs in the BM of leukemia. In the present study, we demonstrate that functional CAFs are widespread in the BM of AML patients and could serve as a critical chemo-protective element for AML cells by producing an abundance of GDF15.

As we all know, AML cells interact both anatomically and functionally with the stroma within the BM microenvironment. These interactions have a critical role in the development, progression and relapse of AML. A recent study has suggested discoidin domain receptor 1 (DDR1), a class of collagen-activated receptor tyrosine kinase (RTK) was highly upregulated on bone marrow (BM)-derived CD33+ leukemic blasts of acute myeloid leukemia (AML) patients, suggesting that the remodeled collagen IV in BM microenvironment could modulate the migration and adhesion of myeloid leukemia cells during leukemogenesis [32]. In this study, the RFD and the immunohistochemistry assay were used to detect the

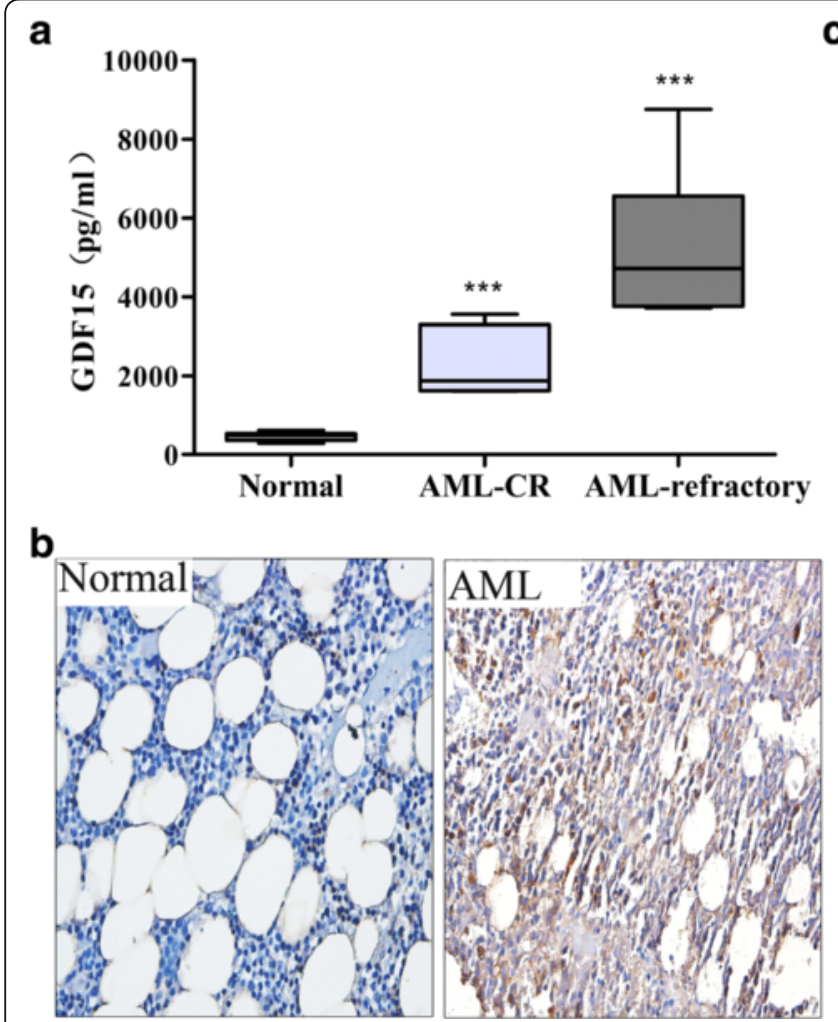

C
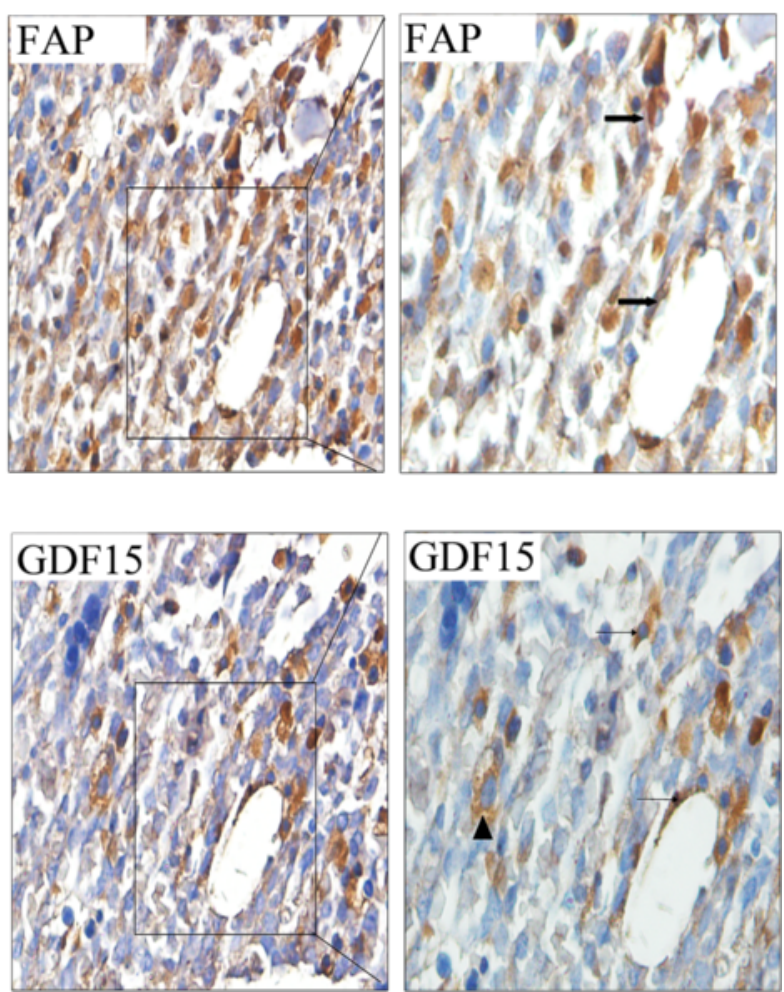

Fig. 5 GDF15 expressed in the BM of AML in vivo. a ELISA analysis of GDF15 levels in the BM aspirates of the controls $(n=11)$ and treatmentnaïve AML-CR $(n=9)$ and AML-refractory patients $(n=8) * * * p<0.001 \mathrm{vs}$ the control group. $\mathbf{b}$ Examples of immunohistochemistry for GDF15 in the BM sections of the normal control and the primary AML patient. Both images are at a magnification of 400x. c Immunohistochemistry identifies cells that express FAP (arrows) and GDF15 (arrowheads) in the AML patients. GDF15 could also be detected in the leukemia cells (triangle). The images on the left are at a magnification of $400 x$ and on the right are at a magnification of $1000 x$ 
transformation of the stroma niche in BM during acute leukemogenesis. It has been reported that reticulin in $\mathrm{BM}$ is mainly composed of individual fibrils or small bunches of fibrils of type III collagen surrounding a core of type I collagen fibrils [20]. Our experiments also confirmed the presence of the amounts of collagen I and collagen III in the marrow of AML (Additional file 1: Figures S3). This result is in agreement with those from previous studies, which have revealed that CAFs might produce and secrete various extracellular matrix proteins (i.e., collagen I, III, IV) in solid tumors [33]. Data has been proposed that CAFs are responsible for collagen synthesis in the stroma of human hepatocellular carcinoma [34]. But reports about the origin of the fiber is still rare, to our knowledge, Jean Y P et al. firstly developed the quantitative dissection of stromal cell-extracellular matrix interactions in living tissue by using multiphoton laser scanning microscopy and second harmonic generation (SHG) of fibrillar collagen. They showed that the CAF is necessary for fiber remodeling [35]. Based on the support from the literatures, we considered that the increased fiber stroma in BM might imply the increased of CAFs during leukemogenesis. Positive staining for the CAF-specific markers $\alpha$-SMA, FAP and FSP1 could also displayed that amount of the CAFs contributed to the abundant fibers in the BM. In addition, there existed sufficient TGF- $\beta 1$ in BM microenvironment of leukemia to stimulate the formation of CAF niche (Additional file 1: Figure S4).

Resistance to anti-cancer therapies which is the major obstacle to a better prognosis of patients obtained increasingly attention in the modern biomedical research [36]. Acting on cancer cells, CAFs have previously been reported to be involved in resistance to chemotherapy in many solid tumors. Our co-cultured experiments showed that these functional fibroblasts could also exhibit a chemo-protective effect on the leukemia cells. It is worth noting that our results did no find the similar protective effects of the stroma cells which were derived from the healthy donors and pretreated with TGF$\beta 1(10 \mathrm{ng} / \mathrm{ml})$ for $14 \mathrm{~d}$ (Additional file 1: Figure S5). We suspected this may be due to the differences in BMMSC themselves between the AML patients and the healthy ones. The mechanisms involved in the antichemotherapy effects of CAFs on AML cells are not clear. In this report, we detected that either knockdown of GDF15 in the CAFs or interference by neutralizing anti-GDF15 in the co-culture system decreased the mortality of the THP-1 cells, confirming that GDF15 has an essential function in the CAF-mediated chemotherapeutic resistance of the leukemic cells. When compared with MSC and the THP-1 cells, we detected an increased GDF15 concentration in the conditioned medium of the CAFs (Additional file 1: Figure S6). Therefore, we assumed that CAFs were likely to be the main contributor to the high concentrations of soluble GDF15, which was different form our previous publication that GDF15 derived from leukemia cells conferred chemoresistance in acute lymphoblastic leukemia, we suspect there existed originally differences in the lymphoid and myeloid leukemia cells. As we all know that GDF15, as an important component of the TGF- $\beta$ superfamily, is notably increased in patients with prostate, colorectal, or pancreatic cancers and has been described as an important biomarker of poor clinical outcome [37-39]. However, the GDF15 concentrations in the BM of AML patients are not well defined. In our experiments, the direct evidence for the CAFs contributing to high GDF15 expression is supported by the BM biopsies, which indicated that this factor was mainly expressed by the CAFs and colocalized with FAP by immunohistochemistry. GDF15 is present both on the membrane and in the cytoplasm of leukemic cells (Fig. 5c), suggesting that leukemia cells have an autocrine function, which is consistent with the results of ELISA analysis of the leukemia cell lines in vitro. Both the CAFs and the leukemia cells secreted GDF15, suggesting that GDF15 plays a critical role in the local cross-regulation between the leukemia cells and the CAFs within the BM in primary AML patients. However, the detection of CAF cells on BM biopsies were not routinely performed in AML patients, so if there resist a correlation between CAF density and AML survival still remains unclear. Based on this, it is necessary for us to construct the mouse transplantation models of AML to explore the correlation between CAF density and further to explore the potential mechanisms in vivo.

\section{Conclusions}

This study highlights the importance of the fiber stroma in leukemogenesis. The abundant reticulin fiber predicts a poor outcome of AML. Based on the experiments, we suggest that a portion of the CAF cells exist in the BM microenvironment of AML. And these functional cells affected the chemosensitivity of the leukemia cells by producing GDF15. Disrupting GDF15 via treatment with an anti-GDF15 antibody or si-GDF15 in the CAFs abrogated the protection of the leukemic cells by CAFs. To our knowledge, this is the first report of the molecular mechanisms of CAFs in AML. We suspect that there might maintain a chemo-protective niche in a pool of AML cells causes the relapse, which may need for further animal experiments to verification. In this regard, future studies from our laboratory will assess GDF15 as a novel target for therapeutic strategies in AML in vivo by using a mouse transplantation model of AML. 


\section{Additional file}

Additional file 1: Figure S1. Phenotype characterization of BMMSCs. Flow Cytometry analysis showed that almost all the cultured MSC expressed CD90, CD105 and CD73, while a small portion of MSC expressed CD14, CD34 and CD45. Figure S2. Differentiation potential of BMMSCs. (a) MSC differentiation to adipocytes was shown by Oil-O-Red staining. (B) And osteoblast differentiation was detected by Alizarin Red staining. The images are at a magnification of 200x. Figure S3. Immunohistochemistry to detect the expression of collagen I and collagen III in the BM of the normal control and the AML patient. Both images are at a magnification of 400X.

Figure S4. The levels of TGF- $\beta 1$ in BM plasma between AML patients and the normal controls were analyzed by the Quantikine ELISA kit ((R\&D Systems, USA), according to the manufacturers instruction. The absorbance at $450 \mathrm{~nm}$ was detected by the microplate reader. Concentrations were calculated from the constructed linear curve. Data are presented as the mean \pm standard deviation; $n=5$ per group. ${ }^{* *} p<0.01$. Figure S5. Bar plots illustrating the viability of leukemia cells (THP-1 and K562) under treatment of Ara-C (10uM) for $48 \mathrm{~h}$. THP-1/K562 cells were cultured in medium alone or co-cultured with the stroma cells derived from BM-MSC of healthy donors and pretreated with TGF- $\beta 1(10 \mathrm{ng} / \mathrm{ml})$ or not. The error bars represent the standard error of the mean of three replicates, ns, $p>0.05$. Figure $\mathbf{S 6}$. The horizontal bar showed Elisa analysis of GDF15 in the supernatants of the MSC, THP and CAF cells. The data represent the mean \pm SD of triplicate experiments, ${ }^{* *} p<0.01$. (DOC $6094 \mathrm{~kb}$ )

\section{Acknowledgements}

This study was supported by the National Natural Science Foundation of China (Grant No. 81570135), National Science and Technology Major Equipment Projects of China (Grant No.2013YQ03065109) and the Shanghai Committee of Science and Technology, China (Grant No.13D22293700).

\section{Authors' contributions}

YZ performed the experiments, analyzed the results and wrote the manuscript. JZ and HW performed the IHC and GO staining. WL carried out the siRNA transfection analysis. SL, YY, WW, and ZD collected patient samples and analyzed clinical information. QZ providing clinical samples and JS designed the experiments and wrote the paper. All authors read and approved the final version of the submitted manuscript.

\section{Competing interests}

The authors declare that they have no competing interests.

\section{Author details}

'Department of Hematology, Shanghai Jiao Tong University Affiliated Sixth People's Hospital, Shanghai 200233, China. ²Department of Hematology, Tongren Hospital, Shanghai Jiao Tong University School of Medicine, Shanghai 200336, China. ${ }^{3}$ Department of Hematology, Shanghai Jiao Tong University Affiliated Sixth People's Hospital South campus, Shanghai 201400, China. ${ }^{4}$ Department of Hematology, Shanghai Jiao Tong University School of Medicine Affiliated Ninth People's Hospital, Shanghai 200011, China.

Received: 2 February 2016 Accepted: 11 August 2016 Published online: 19 September 2016

\section{References}

1. Ayala F, Dewar R, Kieran M, et al. Contribution of bone microenvironment to leukemogenesis and leukemia progression. Leukemia. 2009;23(12):2233-41.

2. Valcz G, Sipos F, Tulassay Z, et al. Importance of carcinoma-associated fibroblastderived proteins in clinical oncology. J Clin Pathol. 2014;67(12):1026-31.

3. Polanska UM, Orimo A. Carcinoma-associated fibroblasts: non-neoplastic tumour-promoting mesenchymal cells. J Cell Physiol. 2013;228(8):1651-7.

4. $\quad$ Li Q, Zhang D, Wang Y, et al. MiR-21/Smad7 signaling determines TGF- $\beta 1$ induced CAF formation. Sci Rep. 2013;3:2038.

5. Gu J, Qian H, Shen L, et al. Gastric cancer exosomes trigger differentiation of umbilical cord derived mesenchymal stem cells to carcinoma-associated fibroblasts through TGF- $\beta / S m a d$ Pathway. PLoS One. 2012;7(12):e52465.

6. Peng Q, Zhao L, Hog Y, et al. Biological characteristics and genetic heterogeneity between carcinoma-associated fibroblasts and their paired normal fibroblasts in human breast cancer. PLoS One. 2013;8(4):e60321.
7. Bruzzese F, Hägglöf $C$, Leone $A$, et al. Local and systemic protumorigenic effects of cancer-associated fibroblast-derived GDF15. Cancer Res. 2014; 74(13):3408-17.

8. Ostman A, Augsten M. Cancer-associated fibroblasts and tumor growthbystanders turning into key players. Curr Opin Genet Dev. 2009;19(1):67-73.

9. De Veirman K, Rao L, De Bruyne E, et al. Cancer Associated Fibroblasts and Tumor Growth: Focus on Multiple Myeloma. Cancers (Basel). 2014;6(3):1363-81.

10. Duan CW, Shi J, Chen J, et al. Leukemia Propagating Cells Rebuild an Evolving Niche in Response to Therapy. Cancer Cell. 2014;25(6):778-93.

11. Breit SN, Johnen $H$, Cook AD, et al. The TGF- $\beta$ superfamily cytokine, MIC-1/ GDF15: a pleotrophic cytokine with roles in inflammation, cancer and metabolism. Growth Factors. 2011;29(5):187-95.

12. Roth $\mathrm{P}$, Junker $M$, Tritschler l, et al. GDF-15 contributes to proliferation and immune escape of malignant gliomas. Clin Cancer Res. 2010;16(15):3851-9.

13. Wang $X$, Li Y, Tian H, et al. Macrophage inhibitory cytokine 1 (MIC-1/GDF15) as a novel diagnostic serum biomarker in pancreatic ductal adeno carcinoma. BMC Cancer. 2014;14:578.

14. Bock AJ, Stavnes HT, Kempf T, et al. Expression and Clinical Role of Growth Differentiation Factor-15 in Ovarian Carcinoma Effusions. Int J Gynecol Cancer. 2010;20(9):1448-55.

15. Corre J, Labat E, Espagnolle $\mathrm{N}$, et al. Bioactivity and prognostic significance of growth differentiation factor GDF15 secreted by bone marrow mesenchymal stem cells in multiple myeloma. Cancer Res. 2012;72(6):1395-406.

16. Cheson BD, Bennett JM, Kopecky KJ, et al. Revised recommendations of the International Working Group for Diagnosis, Standardization of Response Criteria, Treatment Outcomes, and Reporting Standards for Therapeutic Trials in Acute Myeloid Leukemia. J Clin Oncol. 2003;21(24):4642-9.

17. Schmid C, Schleuning M, Schwerdtfeger R, et al. Long-term survival in refractory acute myeloid leukemia after sequential treatment with chemotherapy and reduced-intensity conditioning for allogeneic stem cell transplantation. Blood. 2006;108(3):1092-9.

18. Norén-Nyström $U$, Roos $G$, Bergh $A$, et al. Bone marrow fibrosis in childhood acute lymphoblastic leukemia correlates to biological factors, treatment response and outcome. Leukemia. 2008;22(3):504-10.

19. Nath SV, Nicholson I, Tapp H, et al. Reticulin fibres anchor leukaemic blasts in the marrow of patients with acute lymphoblastic leukaemia. Med Hypotheses. 2011;77(3):333-5.

20. Kuter DJ, Bain B, Mufti G, et al. Bone marrow fibrosis: pathophysiology and clinical significance of increased bone marrow stromal fibres. Br J Haematol. 2007:139(3):351-62.

21. Papadantonakis N, Matsuura S, Ravid K. Megakaryocyte pathology and bone marrow fibrosis: the lysyl oxidase connection. Blood. 2012;120(9):1774-81.

22. Le Bousse-Kerdilès MC, Martyré MC, Samson M. Cellular and molecular mechanisms underlying bone marrow and liver fibrosis: a review. Eur Cytokine Netw. 2008;19(2):69-80

23. Calon A, Tauriello DV, Batlle E. TGF-beta in CAF-mediated tumor growth and metastasis. Semin Cancer Biol. 2014;25:15-22.

24. Griner SE, Wang KJ, Joshi JP, et al. Mechanisms of Adipocytokine-Mediated Trastuzumab Resistance in HER2-Positive Breast Cancer Cell Lines. Curr Pharmacogenomics Person Med. 2013;11(1):31-41.

25. Meier JC, Haendler B, Seidel $H$, et al. Knockdown of platinum-induced growth differentiation factor 15 abrogates p27-mediated tumor growth delay in the chemoresistant ovarian cancermodel A2780cis. Cancer Med. 2015;4(2):253-67.

26. Mimeault M, Johansson SL, Batra SK, et al. Marked improvement of cytotoxic effects induced by docetaxel on highly metastatic and androgenindependent prostate cancer cells by downregulating macrophage inhibitory cytokine-1. Br J Cancer. 2013;108(5):1079-91.

27. Huang CY, Beer TM, Higano CS, et al. Molecular alterations in prostate carcinomas that associate with in vivo exposure to chemotherapy: identification of a cytoprotective mechanism involving growth differentiation factor 15. Clin Cancer Res. 2007;13(19):5825-33.

28. Alexeyenko A, Alkasalias T, Pavlova T, et al. Confrontation of fibroblasts with cancer cells in vitro: gene network analysis of transcriptome changes and differential capacity to inhibit tumor growth. J Exp Clin Cancer Res. 2015;34(1):62.

29. McDonald LT, Russell DL, Kelly RR, et al. Hematopoietic stem cell-derived cancer-associated fibroblasts are novel contributors to the pro-tumorigenic microenvironment. Neoplasia. 2015;17(5):434-48.

30. Cheng $Y$, Wang $K, M a ~ W$, et al. Cancer-associated fibroblasts are associated with poor prognosis in esophageal squamous cell carcinoma after surgery. Int J Clin ExpMed. 2015;8(2):1896-903. 
31. Sukowati CH, Anfuso B, Crocé $\mathrm{LS}$, et al. The role of multipotent cancer associated fibroblasts in hepatocarcinogenesis. BMC Cancer. 2015;30(18-1):188.

32. Amanda J F, Vary CPH, Brooks PC, et al. Cryptic collagen IV promotes cell migration and adhesion in myeloid leukemia. Cancer Med. 2014;3(2):265-72.

33. Valcz G, Sipos F, Tulassay Z, et al. Republished: Importance of carcinomaassociated fibroblast-derived proteins in clinical oncology. Postgrad Med J. 2015;91(1075):1026-31.

34. Faouzi S, Le Bail B, Neaud V, et al. Myofibroblasts are responsible for collagen synthesis in the stroma of human hepatocellular carcinoma: an in vivo and in vitro study. J Hepatol. 1999;30(2):275-84.

35. Perentes JY, McKee TD, Ley $C D$, et al. In vivo imaging of extracellular matrix remodeling by tumor-associated fibroblasts. Nat Methods. 2009;6(2):143-5.

36. Niero EL, Rocha-Sales B, Lauand C, et al. The multiple facets of drug resistance one history, different approaches. J Exp Clin Cancer Res. 2014;33(1):37.

37. Brown DA, Lindmark F, Stattin P, et al. Macrophage inhibitory cytokine 1: a new prognostic marker in prostate cancer. Clin Cancer Res. 2009;15(21):6658-64.

38. Wallin U, Glimelius B, Jirström K, et al. Growth differentiation factor 15: a prognostic marker for recurrence in colorectal cancer. Br J Cancer. 2011; 104(10):1619-27.

39. Koopmann J, Rosenzweig CN, Zhang Z, et al. Serum markers in patients with resectable pancreatic adenocarcinoma: macrophage inhibitory cytokine 1 versus CA19-9. Clin Cancer Res. 2006;12(2):442-6.

Submit your next manuscript to BioMed Central and we will help you at every step:

- We accept pre-submission inquiries

- Our selector tool helps you to find the most relevant journal

- We provide round the clock customer support

- Convenient online submission

- Thorough peer review

- Inclusion in PubMed and all major indexing services

- Maximum visibility for your research

Submit your manuscript at www biomedcentral.com/submit

\section{(O) BioMed Central}

